\title{
FATORES QUE INFLUENCIAM A RENTABILIDADE DAS INSTITUIÇÕES FINANCEIRAS BANCÁRIAS BRASILEIRAS: CONCLUSÕES COM BASE NA MODELAGEM DE EQUAÇÕES ESTRUTURAIS
}

\author{
FACTORS THAT INFLUENCE THE PROFITABILITY OF BRAZILIAN BANKING \\ INSTITUTIONS: CONCLUSIONS BASED ON THE MODELING OF STRUCTURAL \\ EQUATIONS
}

\section{FACTORES QUE INFLUENCIAR LA RENTABILIDAD DE LAS INSTITUCIONES FINANCIERAS BANCARIAS BRASILEÑAS: CONCLUSIONES CON BASE EN EL MODELADO DE ECUACIONES ESTRUCTURALES}

Douglas José Mendonça, Programa de PósGraduação em Administração (UFLA), Lavras, MG, Brasil

Júlia Alves e Souza, Programa de PósGraduação em Administração (UFLA), Lavras. MG, Brasil

Eduardo Gomes Carvalho, Centro Federal de Educação Tecnológica de Minas Gerais (CEFET-MG), Vargínia, MG, Brasil.

Francisval de Melo Carvalho, Universidade Federal de Lavras (UFLA),

Lavras, MG, Brasil

Artigo Submetido em 18.12.2017. Aprovado em 16.02.2018.

Avaliado pelo processo de double blind review

e-mail para contato: mendonca_douglas@yahoo.com.br
RESUMO

Este estudo objetivou identificar fatores que influenciam a rentabilidade das instituições financeiras bancárias no Brasil.A pesquisa propôs a utilização da Modelagem de Equações Estruturais (SEM) para investigar tais fatores. Aamostrafoi composta por150 instituições financeiras bancárias brasileiras, no período de 2012 a 2016. Para a análise dos dados, foi utilizada a SEMcom o modelo de medição reflexivo. As variáveis empregadas para representar a rentabilidade foram o Retorno sobre Ativos (ROA) e o Retorno sobre o Patrimônio Líquido (ROE). Foram testadas 5 hipóteses, referentes às variáveis macroeconômicas, ao tamanho das instituições, à captação, à diversificação e às despesas operacionais. No modelo de mediação reflexiva ajustado no estudo, todos os critérios de avaliação foram atendidos, fornecendo suporte para a confiabilidade e validade das medidas reflexivas. Como resultados, observou-se que as variáveis latentes testadas explicam, de forma moderada, 20,5\% da variância da rentabilidade. Das 5 hipóteses de pesquisa, 3 foram confirmadas. Concluiu-se que quanto maior a captação (capacidade da instituição de receber depósitos à vista), maior é a rentabilidade da instituição. A diversificação das fontes de receita também está positivamente relacionada à rentabilidade. Por outro lado, ficou evidente que quanto maiores são os gastos operacionais relativamente ao porte do banco, menos rentável é a instituição. Destaca-se que acaptação se revelou, no modelo analisado, como o fator mais importante para explicar a rentabilidade das instituições financeiras bancárias brasileiras.

Palavras chave: Instituições Financeiras, Rentabilidade, Modelagem de Equações Estruturais.

\section{ABSTRACT}

This study aimed to identify factors that influence the profitability of banking financial institutions in Brazil. The research proposed the use of Structural Equation Modeling (SEM) to investigate such factors. The sample consisted of 150 Brazilian banking financial institutions, from 2012 to 2016. For the analysis of the data, the SEM was used with the reflective measurement model. The variables used to represent the profitability were Return on Assets (ROA) and Return on Equity (ROE). Five hypotheses were tested, regarding the macroeconomic variables, the size of institutions, funding, diversification and operational expenses. In the reflexive 


\section{(9)REUNIR}

mediation model adjusted in the study, all evaluation criteria were met, providing support for the reliability and validity of reflexive measures. As results, it was observed that the latent variables tested explain, moderately, 20.5\% of the profitability variance. Of the 5 research hypotheses, 3 were confirmed. It was concluded that the higher the funding (the institution's ability to receive demand deposits), the greater the institution's profitability. The diversification of revenue sources is also positively related to profitability. On the other hand, it became clear that the greater the operational expenses relative to the size of the bank, the less profitable the institution. It should be noted that, in the analyzed model, funding was the most important factor in explaining the profitability of Brazilian banking institutions.

Keywords: Financial Institutions, Profitability, Structural Equation Modeling.

\section{RESUMEN}

Este estudio tuvo el objetivo de identificar factores que influencian la rentabilidad de las instituciones financieras bancarias en Brasil. La investigación propuso la utilización del modelado de ecuaciones estructurales (SEM) para investigar tales factores. La muestra fue compuesta por 150 instituciones financieras bancarias brasileñas, en el período de 2012 a 2016. Para el análisis de los datos, se utilizó la SEM con el modelo de medición reflexivo. Las variables empleadas para representar la rentabilidad fueron el Retorno sobre Activos (ROA) y el Retorno sobre el Patrimonio Líquido (ROE). Se probaron cinco hipótesis, referentes a las variables macroeconómicas, al tamaño de las instituciones, a la captación, a la diversificación ya los gastos operativos. En el modelo de mediación reflexiva ajustado en el estudio, todos los criterios de evaluación fueron atendidos, proporcionando soporte para la confiabilidad y validez de las medidas reflexivas. Como resultados, se observó que las variables latentes probadas explican, de forma moderada, el 20,5\% de la varianza de la rentabilidad. De las 5 hipótesis de investigación, 3 fueron confirmadas. Se concluyó que cuanto mayor sea la captación (capacidad de la institución de recibir depósitos a la vista), mayor es la rentabilidad de la institución. La diversificación de las fuentes de ingresos también está positivamente relacionada con la rentabilidad. Por otro lado, quedó evidente que cuanto mayores son los gastos operativos en cuanto al porte del banco, menos rentable es la institución. Se destaca que la captación se reveló, en el modelo analizado, como el factor más importante para explicar la rentabilidad de las instituciones bancarias brasileñas.

Palabras clave: Instituciones Financieras, Rentabilidad, Modelado de Ecuaciones estructurales.

\section{INTRODUÇÃO}

As instituições financeiras bancárias possuem a função de captar os recursos de agentes econômicos com excesso e os repassar para agentes econômicos com carência; por essa intermediação, tais instituições pagam e recebem os respetivos juros (CARVALHO; RIBEIRO, 2016). De acordo com Akinkunmi (2017), o papel dos bancosé central no financiamento da atividade econômica. Considerando esse aspecto, o estudo dos fatores que influenciam a rentabilidade bancária tem despertado o interesse de pesquisadores, bem como de agentes relacionados àadministração dos bancos, aos mercados financeiros e aos agentes reguladores. 


\section{(9)REUNIR}

Primo et al. (2013) afirmam que o conhecimento dos fatores que influenciam a rentabilidade das instituições financeiras bancárias pode auxiliar no processo de tomada de decisões de depositantes e investidores. Com tal conhecimento, esses agentes poderão estimar os ganhos futuros dos bancos com mais confiabilidade, diante da perspectiva de alterações nos fatores que os determinam.

As instituições financeiras são afetadas por vários fatores internos e externos, que podem ter impactos sobre a rentabilidade bancária (BORIO et al., 2017). Almazari (2014) explica que os fatores internos, como as decisões de gestão, tamanho do banco, captação, gerenciamento de riscos e gerenciamento de despesas, afetam a rentabilidade. Já os fatores externos que afetam a rentabilidade dos bancos, segundo o mesmo autor, estão relacionados a fundamentos institucionais e situações econômicas (ambiente macroeconômico, envolvendo elementos como inflação e taxas de juros).

Para investigar os fatores que influenciam a rentabilidade bancária, a literatura científica tem utilizado predominantemente técnicas estatísticas da primeira geração. Conforme Hair Jr. et al. (2014), essas técnicas envolvem métodos estatísticos que incluem abordagens baseadas em regressão (regressão múltipla, regressão logística e análise de variância) e também técnicas como análise fatorial exploratória, análise de agrupamentos e dimensionamento multidimensional.

No entanto, muitos pesquisadores têm recorrido cada vez mais às técnicas da segunda geração para superar as fraquezas dos métodos da primeira geração (NASCIMENTO; MACEDO, 2016). Entre as técnicas estatísticas da segunda geração, encontra-se a Modelagem de Equações Estruturais (SEM - Structural Equation Modelling). A SEM é uma técnica estatística multivariada que permite avaliar, simultaneamente, relações entre múltiplos constructos; dessa forma, é mais eficiente para a análise de uma série de equações múltiplas (HAIR JR.et al., 2014).

No intuito de ampliar as discussões sobre a rentabilidade bancária trazendo a utilização de uma técnica estatística da segunda geração, esse estudo procura responder à seguinte questão de pesquisa: quais fatores influenciam a rentabilidade das instituições financeiras bancárias no Brasil? Diante disso, o estudo tem como objetivo identificar, por meio da SEM, alguns fatores que influenciam a rentabilidade das instituições financeiras bancárias brasileiras.

Quando comparada à regressão múltipla, a SEM goza de uma variedade de vantagens, como: suposições mais flexíveis; capacidade de analisar múltiplos relacionamentos simultaneamente; trabalhar com variáveis latentes; analisar dados de séries temporais; capacidade de testar os dados não normais; testar modelos com grande número de equações como um todo, obtendo medidas globais de ajuste; capacidade de modelar as variáveis mediadoras e moderadoras; capacidade de modelar termos de erro, entre outras (NASCIMENTO; MACEDO, 2016).

Considerando essas vantagens, o presente estudo pretende contribuir para o avanço do conhecimento na área ao propor a utilização da SEM com o propósito de investigar os fatores que influenciam a rentabilidade bancária. Por meio da Modelagem de Equações Estruturais, são esperados resultados mais robustos e novas contribuições de análise. Por exemplo, pode-se efetuar a avaliação do tamanho do efeito, que permite não apenas identificar a relação entre as variáveis, mas também a contribuição de cada variável do modelo para a rentabilidade bancária. 


\section{QREUNIR}

Este estudo está estruturado em cinco seções, incluindo esta introdução. A segunda seção apresenta o referencial teórico e breve revisão de literatura. Na terceira seção, descreve-se a metodologia empregada. A seção quatro traz a descrição e análise dos resultados obtidos na pesquisa, enquanto que a quinta seção apresenta as considerações finais e as recomendações para futuras pesquisas.

\section{REFERENCIAL TEÓRICO}

\subsection{Potenciais Fatores que Influenciariam a Rentabilidade Bancária}

Para investigar características que influenciariam a rentabilidade bancária, as pesquisas científicas têm se concentrado em fatores específicos da gestão bancária e em fatores macroeconômicos (FRANCIS, 2013).Trujillo-Ponce (2013) complementa que os fatores que determinam a rentabilidade dos bancos podem ser segregados em dois grupos principais. Em primeiro lugar, encontra-se o grupo de fatores que são específicos para cada banco (a estrutura de ativos, a captação, a estrutura financeira, a eficiência, o tamanho e a diversificação de receitas)e que, em muitos casos, são o resultado direto de decisões gerenciais. Já o segundo grupo inclui fatores referentes à estrutura do setor e ao ambiente macroeconômico dentro do qual o sistema bancário opera, tais como a concentração da indústria, a inflação e as taxas de juros.

A modernização do processo de intermediação financeira e a adaptação aos avanços da Tecnologia da Informação (TI) impuseram a necessidade de promover ajustes nos processos operacionais dos bancos. Conforme Wheelock e Wilson (2013), essa necessidade é traduzida em ações voltadas a reduzir a assimetria informacional, diminuir os custos de captação da informação, proporcionar aos clientes maior satisfação e tornar as instituições financeiras mais competitivas, com ganhos de rentabilidade a longo prazo.

Hakenes e Schnabel (2011)e Trujillo-Ponce (2013) argumentam sobre as razões para supor que um banco com maior captação deve apresentar maior rentabilidade. Segundo esses autores, um banco com baixo índice de captação (ou seja, baixo nível de recursos obtidos via depósitos à vista) tende a apresentar maiores custos com o processo de intermediação, uma vez que precisa captar recursos por meio de outras fontes que possuem taxas de juros mais altas do que as praticadas com os depósitos à vista. Assim, um aumento no índice de captação tende a reduzir as despesas com juros sobre dívidas, aumentando a rentabilidade.

Em relação ao tamanho das instituições e seu impacto sobre a rentabilidade, Borio et al. (2017) discutem que existe um consenso na literatura ao considerar que o efeito do tamanho pode ser não linear, com a rentabilidade aumentando inicialmente (conforme aumenta o tamanho) e depois declinando. Se, por um lado, um tamanho maior pode implicar economias de escala, por outro lado os bancos maiores e mais diversificados são mais complexos de serem administrados, o que sugere que os bancos menores podem reduzir de forma mais eficiente os problemas de assimetria de informação associados aos empréstimos.

Também se observa que os fatores macroeconômicos podem influenciar na rentabilidade bancária. Trujillo-Ponce (2013) argumenta que condições econômicas desfavoráveis podem piorar a qualidade da carteira de crédito, gerando perdas de crédito e 


\section{(9REUNIR}

aumentando as provisões que os bancos precisam manter, o que reduziria a rentabilidade. Em contrapartida, uma melhoria nas condições econômicas, além de melhorar a solvência dos clientes, aumentaria a demanda por crédito tanto das famílias como das empresas, produzindo efeitos positivos sobre a rentabilidade dos bancos.

Finalmente, vale destacar que um setor bancário rentável está mais preparado para resistir a choques negativos e contribuir para a estabilidade do sistema financeiro (PRIMO et al., 2013). Diante disso, o conhecimento dos fatores que influenciam a rentabilidade dessas instituições pode auxiliar na tomada de decisão de depositantes e investidores, além de subsidiar os órgãos formuladores da política econômica.

\subsection{Estudos Internacionais sobre Determinantes da Rentabilidade Bancária}

$\mathrm{Na}$ literatura internacional, identificam-se trabalhos que investigaram os fatores que influenciam a rentabilidade bancária. Dentre estes, destacam-se estudos como o de Naceur e Omran (2011), queexaminou a influência da regulação bancária, da concentração e do desenvolvimento financeiro e institucional na rentabilidade em uma ampla seleção de países do Oriente Médio e Norte da África. Foram analisados os anos de 1988 a 2005, e empregouse a metodologia de regressão linear com dados em painel Dinâmico. Os resultados indicaram que a captação bancária e o risco de crédito têm impacto positivo na rentabilidade. Em relação aos indicadores de desenvolvimento macroeconômico, a inflação apresentou uma associação positiva com a rentabilidade.

Já trabalho de Trujillo-Ponce (2013) utilizou a metodologia de regressão com dados em painel para analisar os fatores que determinaram a rentabilidade dos bancos espanhóis no período de 1999-2009. Os resultados demonstraram que a rentabilidade desses bancos esteve associada a uma alta proporção de depósitos à vista (captação), boa eficiência na gestão das despesas operacionais e baixo índice de ativos duvidosos.

Com uma abordagem semelhante, Almazari (2014) utilizou a correlação de Pearson, a análise descritiva da variância e a análise de regressão para investigar os fatores internos que afetam a rentabilidade dos bancos. O principal objetivo foi comparar a rentabilidade dos bancos sauditas e jordanianos entre os anos de 2005 a 2011. Os resultados encontrados indicaram que existe uma correlação positiva da rentabilidade com o tamanho, com a captação e com a liquidez dos bancos analisados.

O estudo de Carvalho e Ribeiro (2016) analisou as instituições financeiras que operaram em Portugal entre os anos de 2002 a 2012. Também foi utilizada a metodologia de regressão com dados em painel. Os resultados sugeriram que a concentração e a captação são significativas na explicação da rentabilidade bancária.

Por fim, ressalta-se que Akinkunmi (2017)utilizou a regressão com dados em painel para investigar os determinantes da rentabilidade dos bancos na Nigéria entre os anos 2001 e 2015. Os resultados mostraram que o índice de eficiência, o risco de crédito e a captação foram os principais determinantes da rentabilidade dos bancos no longo prazo. No entanto, a curto prazo, a inflação e o produto interno bruto real afetaram significativamente o nível de desempenho nos bancos comerciais da Nigéria.

Embora os estudos internacionais utilizem diferentes abordagens para investigar os fatores que influenciam a rentabilidade bancária, é possível notar que nenhum desses estudos utilizou técnicas estatísticas da segunda geração. A maioria dos trabalhos aplicou a 


\section{(9)REUNIR}

regressão linear com dados em painel, que apresenta desvantagens se comparada a técnicas da segunda geração como a SEM.

\subsection{Estudos Nacionais sobre Determinantes da Rentabilidade Bancária}

Na literatura nacional, também são identificadas contribuições anteriores para o entendimento dos fatores determinantes da rentabilidade bancária. O estudo de Rover, Tomazzia e Fávero (2011) investigaram quais fatores econômico-financeiros e macroeconômicos explicam a rentabilidade do setor bancário brasileiro. O período analisado foi do primeiro semestre de 1995 ao segundo de 2009. Foram utilizadas as técnicas de análise de correspondência (ANACOR) e de análise de regressão com dados em painel. Os resultados do estudo evidenciaram que as variáveis econômico-financeiras referentes à liquidez, risco de crédito, despesa operacional, eficiência operacional e alavancagem são relevantes para explicar a rentabilidade bancária brasileira. As condições macroeconômicas concernentes à atividade econômica, inflação e taxa SELIC também foram significativas para explicar tal rentabilidade.

Primo et al. (2013) analisaram os determinantes da rentabilidade bancária no Brasil, tendo como variáveis explicativas fatores econômicos, contábeis e operacionais da atividade bancária. O período analisado foi de 2000 a 2009, e a técnica estatística utilizada foi novamente a regressão com dados em painel. Os resultados demonstraram uma relação positiva da rentabilidade com a taxa básica de juros da economia, com o nível de atividade econômica, com o nível da carga tributária, com a eficiência operacional da instituição e com a participação relativa dos bancos nacionais. Não foram encontrados resultados significativos para o nível de depósitos compulsórios e de encaixe obrigatório.

Já Bittencourt et al. (2017) investigaram, também por meio da regressão com dados em painel, os fatores determinantes para a rentabilidade em bancos múltiplos e cooperativas de crédito no período de 2009 a 2013. Os resultados indicaram que a rentabilidade dessas instituições foi afetada pelas variáveis eficiência, empréstimos, despesas operacionais, captação (depósitos totais), diversificação, taxa Selic e inflação.

Da mesma forma que se pode observar nos estudos internacionais, nos estudos nacionais há uma predominância da utilização de técnicas de análise multivariada da primeira geração (com destaque para a regressão com dados em painel). Evidencia-se, assim, a necessidade da utilização de uma técnica de análise multivariada da segunda geração para investigar os fatores que influenciam a rentabilidade bancária, uma vez que essas técnicas apresentam resultados mais robustos por permitirem identificar a contribuição de cada variável utilizada no modelo para a rentabilidade bancária.

\section{METODOLOGIA}

Nesse tópico, são apresentadas as delimitações da pesquisa, os procedimentos para a definição da amostra e para a coleta de dados. Além disso, são descritas a variáveis utilizadas e as hipóteses de pesquisa, juntamente à apresentação do modelo estrutural proposto. 


\section{(9)REUNIR}

\subsection{Tipo de Pesquisa, Amostra e Coleta de Dados}

Este estudo é caracterizado como quantitativo e explicativo. Conforme Gil (2010), uma pesquisa é considerada quantitativa quando possui uma perspectiva positivista, utilizando-se de uma análise estatística e matemática considerando tudo que pode ser quantificável, traduzindo em números as informações para classificá-las e analisá-las. Já Martins (2002) afirma que a pesquisa explicativa tem como objetivo registrar fatos e identificar suas causas; essa prática visa, por exemplo, ampliar generalizações, definir leis mais amplas, estruturar e definir modelos teóricos, relacionar hipóteses em uma visão mais unitária do universo ou âmbito produtivo em geral. No caso da presente pesquisa, tal perspectiva foi operacionalizada por meio da metodologia quantitativa da Modelagem de Equações Estruturais (SEM).

Hair Jr. et al. (2014) explica que, em um Modelo de Equações Estruturais, é necessário seguir alguns parâmetros para a definição do tamanho da amostra. Os requisitos mínimos de tamanho da amostra necessários para as construções endógenas no modelo estrutural devem assumir um poder estatístico de $80 \%$, com um tamanho do efeito de 0,15 , assumindo um nível de significância de $5 \%$. Para definir o tamanho da amostra da pesquisa seguindo os parâmetros necessários, foi utilizado o software GPower. Tal procedimento levou ao número mínimo de 92 observações a serem analisadas no estudo.

Para a seleção da amostra, foram identificadas, por meio dos registros do Banco Central do Brasil (BCB), as instituições financeiras bancárias com dados disponíveis para os anos de 2012 a 2016 (que corresponde ao período abordado pelo estudo). As instituições que não possuíam dados disponíveis para qualquer um dos anos analisados foram excluídas. Com esse procedimento, a amostra foi formada por 150 instituições (número superior ao mínimo de 92 observações).

Todas as instituições investigadas são classificadas como bancos múltiplos, os quais, segundo Assaf Neto (2003), são organizados sob a forma de sociedade anônima, e devem ter em sua denominação social a expressão "banco". Tais bancos podem ser privados ou públicos e realizam operações ativas, passivas e acessórias (normalmente realizadas por distintos tipos de instituições financeiras).

A pesquisa utilizou dados secundários, obtidos por meio do relatório anual do Banco Central do Brasil - BCB, intitulado "50 Maiores Bancos e o Consolidado do Sistema Financeiro Nacional" disponível no website doBCB (2017). Esse relatório contém os dados consolidados referentes a todas as instituições que compõem o Sistema Financeiro brasileiro. Foram coletados os dados apresentados nas demonstrações contábeis anuais das instituições, englobando o período de 2012 a 2016.

\subsection{Descrição das Variáveis}

A partir dos dados obtidos por meio das demonstrações contábeis correspondentes a cada uma das instituições que compõem a amostra, foram calculados os indicadores econômico-financeiros a elas referentes. As variáveis utilizadas neste estudo para representar a rentabilidade são o Retorno sobre Ativos (ROA) e o Retorno sobre o Patrimônio Líquido (ROE). 


\section{(9REUNIR}

O ROA, calculado pela divisão do lucro líquido pelo ativo total, reflete a capacidade do banco em gerar lucro por meio da utilização do total de recursos aplicados; já o ROE, obtido pela divisão do lucro líquido pelo patrimônio líquido, mensura o retorno que a empresa obtém em relação aos recursos aplicados por seus proprietários ou acionistas (ASSAF NETO, 2012).

As variáveis utilizadas para explicar a rentabilidade dos bancos foram divididas em dois grupos. O primeiro representa os indicadores econômico-financeiros, dos próprios bancos, enquanto o segundo abrange algumas das condições econômicas durante o período analisado. As variáveis referentes aos indicadores são:

- Tamanho dos bancos (TAM): é calculado pelo logaritmo dos ativos totais. É utilizado para medir o ganho em escala ao maximizar a utilização dos fatores de produção.

- Captação (CAP): é calculada pela razão entre o total de depósitos à vista e o total de ativos. Mede a capacidade que uma instituição possui de captar depósito à vista. A instituição que capta um maior volume de depósitos à vista consegue ter um maior montante de capital para a operação de intermediação financeira, a um baixo custo.

- Grau de diversificação (GDI): é calculado pela razão entre as receitas operacionais e o total de ativos. Refere-se às fontes alternativas de receita (que não são provenientes da intermediação financeira), como as receitas com serviços e taxas.

- Indicador de despesa operacional (DOA): é calculado pela razão entre as despesas operacionais e o total de ativos. Refere-se aos gastos operacionais relativamente ao porte do banco.

Já as variáveis macroeconômicas, que representam as condições de mercado utilizadas para explicar a rentabilidade dos bancos, são as seguintes:

- Inflação (Inflação): corresponde à inflação corrente, calculada pela variação anual do IPCA e elaborada pelo Instituto Brasileiro de Geografia e Estatística (IBGE). Foi utilizado o valor médio anual do IPCA para cada ano da amostra.

- Taxa de juros da economia (Selic): trata-se da taxa básica de juros da economia, que consiste no instrumento de juros utilizado como parâmetro pelo Banco Central do Brasil (BCB). Foi utilizada a Taxa Selic média anual para cada ano em estudo (taxa anualizada em pontos percentuais), disponibilizada pelo BCB.

\subsection{Análise dos Dados e Hipóteses de Pesquisa}

Para a análise dos dados, foi utilizada a Modelagem de Equações Estruturais (SEM Structural Equation Modelling). A SEM é um conjunto de técnicas estatísticas multivariadas que possibilitam o exame simultâneo de um conjunto de relacionamentos teóricos entre uma ou mais variáveis independentes, contínuas ou discretas, e uma ou mais variáveis dependentes, também contínuas ou discretas (TABACHNICK; FIDELL, 1996). Segundo Hair Jr. et al. (2014), a SEM capacita o pesquisador a examinar, simultaneamente, múltiplas relações de dependência e independência entre variáveis latentes, por meio de variáveis observadas.

Existem dois tipos de escala de mensuração em SEM: reflexivas ou formativas. $\mathrm{Na}$ abordagem reflexiva, a direção de causalidade parte da variável latente para os indicadores, ou seja, o constructo latente causa os itens observáveis. Já nas escalas formativas, as variáveis latentes são consideradas efeitos em detrimento de causas. Dessa forma, o modelo de medição formativa é um tipo de configuração do modelo de medição em que os 


\section{(9)REUNIR}

indicadores causam o constructo e as "setas" (que representam as relações no modelo) apontam dos indicadores para o constructo (HAIR JR. et al., 2014).

Nesse estudo, o modelo utiliza a medição reflexiva. De acordo com Nascimento e Macedo (2016), essa medição é recomendada na pesquisa em Contabilidade e Finanças, visto que por meio dela é possível identificar as causas de decisões gerenciais ou a explicação dos escores de desempenho medidos em tarefas de decisões simuladas.

Na Figura 1, estão representados o modelo e as hipóteses de pesquisa, que permitem a visão geral das variáveis analisadas.

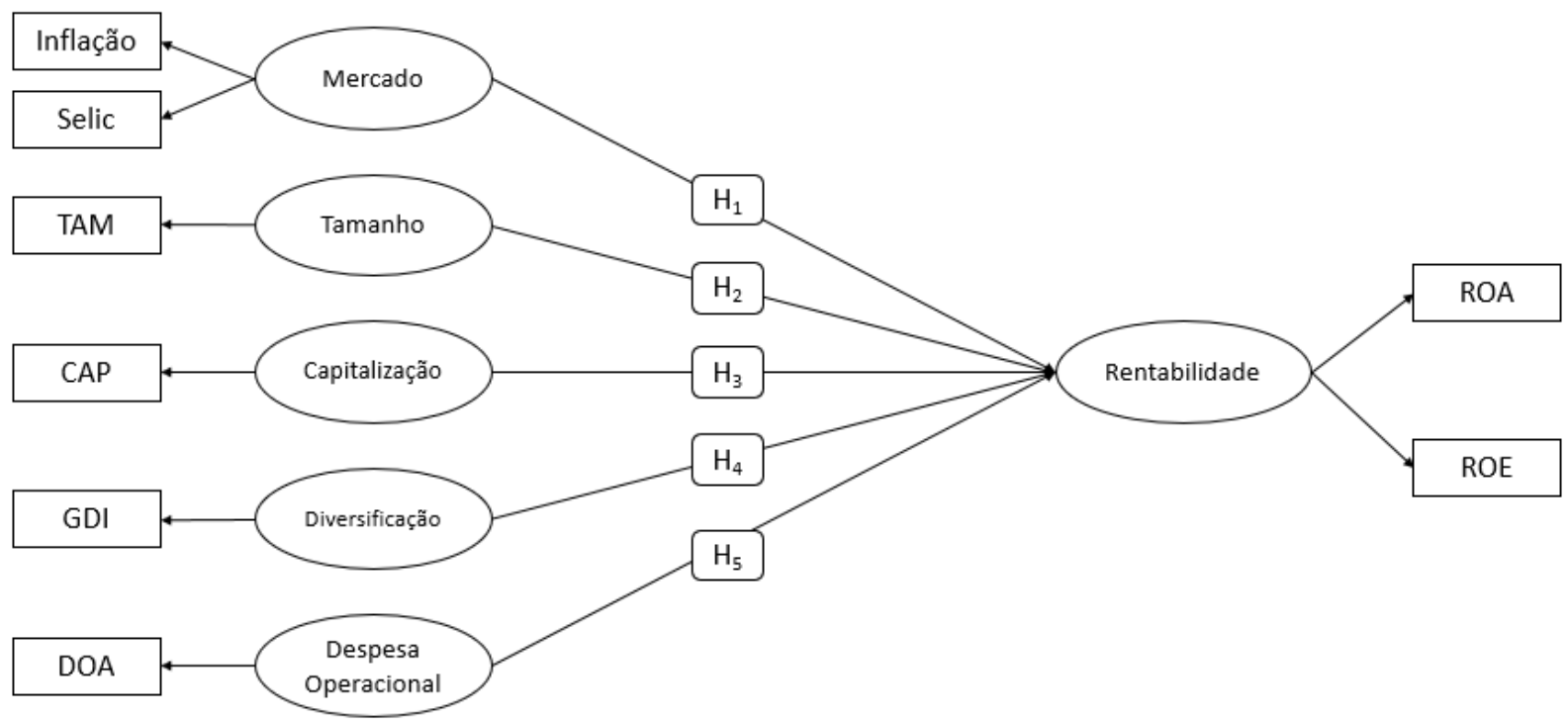

Figura 10 modelo SEM e as hipóteses de pesquisa

Fonte: elaborado pelos autores

O modelo conceitual da pesquisa, representado na Figura 1, engloba as cinco principais hipóteses de pesquisa descritas a seguir:

- $\mathrm{H}_{1}$ : As variáveis macroeconômicas do mercado têm um efeito positivo na rentabilidade das instituições financeiras bancárias no Brasil.

Evidências empíricas demonstram a existência de uma relação positiva entre inflação e rentabilidade, e também indicam que a taxa de juros é positivamente correlacionada com a rentabilidade dos bancos (ROVER et al., 2011).

- $\mathrm{H}_{2}$ : $\mathrm{O}$ tamanho das instituições financeiras bancárias brasileiras tem um efeito positivo sobre a rentabilidade destas.

Essa hipótese supõe que as instituições de maior tamanho tendem a ter maior rentabilidade, tal como abordado nos estudos de Kirkwood e Nahm (2006), Sufian e Majid (2009),e Mendonça et al. (2017). Brasil.

- $\mathrm{H}_{3}$ : A captação tem um efeito positivo na rentabilidade das instituições bancárias no

A captação apresentaria uma relação positiva com a rentabilidade dos bancos porque as instituições financeiras com maiores níveis de depósitos à vista reduzem seus custos de financiamento ao sinalizarem uma menor possibilidade de falência (PASIOURAS; KOSMIDOU, 2007; MENDONÇA et al., 2017).

- $\mathrm{H}_{4}$ : A diversificação tem um efeito positivo na rentabilidade das instituições bancárias no Brasil. 


\section{(9)REUNIR}

A diversificação é referente às fontes alternativas de receita (por exemplo, receitas com serviços e taxas). A literatura tem revelado que quanto maior é o volume de diferentes fontes de receitas, maior tende a ser a rentabilidade dos bancos (KIRKWOOD; NAHM, 2006; SUFIAN;MAJID; 2009).

- $H_{5}$ : As despesas operacionais têm um efeito negativo na rentabilidade das instituições bancárias no Brasil.

As despesas operacionais são referentes aos gastos operacionais para a prestação dos serviços bancários. A literatura indica que quanto maior o volume de despesas assumido pela instituição financeira, menor é a sua rentabilidade (FIORDELISI, 2008; NACEUR; OMRAN 2011; MENDONÇA et al., 2017).

Para investigar as hipóteses de pesquisa e a operacionalizar o Modelo de Equações Estruturais proposto, utilizou-se do software SmartPLS, versão 3.

\section{RESULTADOS E DISCUSSÕES}

Os resultados obtidos foram divididos em duas seções. Na primeira delas, são discutidos os resultados referentes à avaliação do modelo estrutural reflexivo, que é a primeira parte de uma análise que utiliza a Modelagem de Equações Estruturais. Ressalta-se que só é possível tirar conclusões com um modelo estrutural ajustado (se houver falha nos parâmetros de ajuste do modelo, não se pode prosseguir com a análise dos resultados obtidos). Já na segunda seção, é abordada a análise do modelo estrutural e os resultados para as hipóteses de pesquisa testadas.

\subsection{Avaliação do Modelo de Mediação Reflexiva}

A avaliação de um modelo de mediação reflexiva (relações entre os indicadores e constructos) envolve os seguintes elementos: confiabilidade composta (composite reliability), validade convergente (variance extracted), confiabilidade do indicador (indicator reliability) e validade discriminante (discriminant validity). Todos esses elementos devem ser observados para garantir que o modelo esteja ajustado adequadamente.

Os resultados que permitem a avaliação da confiabilidade composta, da validade convergente e da confiabilidade do indicador, para o modelo da presente pesquisa, são demonstrados na Tabela 1.

Tabela 1 Resumo de resultados para o modelo de medição reflexiva ajustado na pesquisa

\begin{tabular}{ccccc}
\hline Variável Latente & Indicadores & Cargas Externas & $\begin{array}{c}\text { Confiabilidade } \\
\text { Composta }\end{array}$ & AVE \\
\cline { 2 - 4 } Rentabilidade & ROA & 0,996 & 0,844 & 0,805 \\
\cline { 2 - 4 } & ROE & 0,786 & 1,00 & 1,00 \\
\hline Captação & CAP & 1,00 & 1,00 & 1,00 \\
\hline Tamanho & TAM & 1,00 & 0,733 & 0,741 \\
\hline Mercado & Inflação & 0,718 & 1,00 \\
\hline Diversificação & Selic & 0,983 & 1,00 & 1,00 \\
\hline
\end{tabular}




\section{(9REUNIR}

Fonte: elaborado pelos autores

A partir dos resultados que constam na Tabela 1 , pode-se obter as seguintes conclusões:

\section{i. Confiabilidade Composta}

Conforme Hair Jr. et al. (2014), a confiabilidade composta é usada para avaliar se a amostra, de fato, está livre de vieses ou ainda se as respostas - em seu conjunto - são confiáveis. Para os autores, os valores de confiabilidade composta entre 0,60 e 0,70 são considerados adequados em pesquisas exploratórias, enquanto que valores de 0,70 e 0,90 são considerados satisfatórios para os demais tipos de pesquisa.

Como apresentado, o menor valor de confiabilidade composta 0,733 é para o constructo Mercado. O constructo Rentabilidade apresentou um valor de 0,844 e os demais constructos apresentaram valor igual a 1,00 , pois são formados por um indicador. Os resultados demonstram que os constructos são confiáveis, visto que todos os valores são superiores a 0,70. Com isso, pode-se concluir pela existência de níveis elevados de consistência interna nas variáveis latentes.

\section{ii. Validade Convergente}

A validade convergente é a extensão em que uma medida se correlaciona positivamente com medidas alternativas do mesmo constructo (NASCIMENTO; MACEDO, 2016). Para estabelecer a validade convergente, são consideradas as cargas externas dos indicadores e a Variância Média Extraída (Average Variance Extracted - AVE).

A AVE é a parcela dos dados das variáveis que é explicada por cada um dos constructos latentes respectivos, ou seja, representa o quanto, em média, as variáveis se correlacionam positivamente com os seus respectivos constructos (RINGLE; SILVA; BIDO, 2014). Fornell e Larcker (1981) explicam que quando as AVE são maiores que 0,50, admite-se que o modelo converge a um resultado satisfatório. Para o modelo ajustado na pesquisa, a AVE de todos os constructos foi superior a 0,50 indicando que o modelo converge para um resultado satisfatório (conclui-se pela existência de validade convergente).

\section{iii. Confiabilidade do Indicador}

A confiabilidade do indicador ocorre quando existem altas cargas externas no mesmo constructo, indicando que os indicadores associados têm muito em comum com o fenômeno captado pelo constructo latente. Hair Jr. et al. (2014) afirma que as cargas externas de todos os indicadores devem ser estatisticamente significativas. Diante disso, espera-se valores padronizados com cargas externas superiores a 0,708. Para o modelo ajustado, todas as cargas externas são superiores a 0,708 (Tabela 1), demonstrando que as cargas externas de todos os indicadores são estatisticamente significativas. Assim, pode-se concluir que são indicadores confiáveis.

Já o quarto e último elemento a ser observado ao se avaliar um modelo de mediação reflexiva é a validade discriminante, que consiste na medida que um constructo é verdadeiramente distinto dos demais constructos em um modelo estrutural (HAIR JR. et al., 2014). Assim, o estabelecimento de validade discriminante implica que o constructo é único e capta fenômenos não compreendidos pelos demais constructos contidos no modelo ajustado.

A forma principal de se avaliar a validade discriminante é por meio da confrontação das raízes quadradas dos valores da AVE de cada constructo frente às correlações de 


\section{(9)REUNIR}

Pearson entre os demais constructos latentes, critério que foi proposto por Fornell e Larcker (1981). A Tabela 2 apresenta os valores quadráticos da AVE de cada constructo e as correlações entre as variáveis latentes obtidos no modelo.

Tabela 2 Resultados para a Validade Discriminante

\begin{tabular}{lcccccc}
\hline & Captação & $\begin{array}{c}\text { Despesa } \\
\text { Operacional }\end{array}$ & Diversificação & $\begin{array}{c}\text { Mercad } \\
\text { o }\end{array}$ & Rentabilidade Tamanho \\
\hline Captação & 1,000 & & & & & \\
\hline Despesa operacional & 0,195 & 1,000 & & & & \\
\hline Diversificação & 0,081 & 0,289 & 1,000 & & & \\
\hline Mercado & 0,044 & 0,034 & 0,087 & 0,861 & & \\
\hline Rentabilidade & 0,399 & $-0,048$ & 0,141 & 0,079 & 0,897 & 1,000 \\
\hline Tamanho & $-0,166$ & $-0,011$ & 0,159 & 0,083 & 0,018 & \\
\hline
\end{tabular}

Fonte: elaborado pelos autores

Segundo Hair Jr. et al. (2014), haverá validade discriminante se as correlações entre as variáveis latentes forem inferiores à raiz quadrada da AVE. A AVE obtida pelas variáveis latentes Rentabilidade e Mercado (0,844 e 0,733 respectivamente) são inferiores aos valores obtidos pela raiz quadrada da AVE, sendo que para a variável latente Rentabilidade o valor da raiz foi de 0,897 e para a variável latente Mercado foi de 0,861 . Os demais constructos são formados por uma variável, por isso não há existência de correlação. Como os valores quadráticos das AVE's de todos os constructos latentes formados por mais de um indicador são superiores aos valores das correlações, pode-se concluir pela existência de validade discriminante.

Diante disso, pode-se afirmar que todos os critérios de avaliação do modelo foram atendidos, fornecendo suporte para a confiabilidade e a validade das medidas reflexivas.

\subsection{Avaliação do Modelo Estrutural}

Após a avaliação do modelo de mensuração, o próximo passo é avaliar o modelo estrutural e verificar se as hipóteses de pesquisa foram satisfeitas. Nesse aspecto, Hair Jr. et al. (2014) explicam que é importante relatar e discutir os coeficientes de determinação $R^{2}$, o tamanho e significância dos coeficientes de caminho, os tamanhos do efeito $f^{2}$ e os tamanhos de efeito $q^{2}$.

Inicialmente, foram verificadas as correlações entre as variáveis do estudo, buscando identificar a existência de possíveis indícios de multicolinearidade. Os resultados da estatística VIF para os indicadores e constructos são demonstrados na Tabela 3.

Tabela 3 Estatísticas de colinearidade (VIF) dos indicadores e dos constructos

\begin{tabular}{lccc}
\hline Variável Latente & Indicadores & VIF indicadores & VIF constructos \\
\cline { 2 - 4 } Rentabilidade & ROA & 2,146 & \\
\hline Captação & ROE & 2,146 & \\
\hline Tamanho & CAP & 1,000 & 1,075 \\
\hline \multirow{2}{*}{ Mercado } & TAM & 1,000 & 1,068 \\
\hline Diversificação & Inflação & 1,503 & 1,015 \\
\cline { 2 - 4 } & Selic & 1,503 & 1,132 \\
\hline
\end{tabular}




\section{(9)REUNIR}

\begin{tabular}{llll}
\hline Despesa operacional & DOA & 1,000 & 1,129 \\
\hline
\end{tabular}

Fonte: elaborado pelos autores

Por meio da Tabela 03, é possível observar que a estatística VIF (fatores de inflação de variância) confirmou a ausência de multicolinearidade. O VIF externo (ou dos indicadores variou entre 1,000 e 2,146, e o VIF interno (ou dos constructos) variou entre 1,015 e 1,132 (valores de VIF menores que 5,0 indicam que não há multicolinearidade).

Avaliando o grau de explicação da variância da variável endógena alvo, no caso a rentabilidade, $0 R^{2}$ foi de 0,205 , o que permite concluir que as cinco variáveis latentes testadas (captação, tamanho, mercado, diversificação e despesa operacional) explicam, de forma moderada, $20,5 \%$ da variância da rentabilidade. Os resultados acerca dos tamanhos e significância dos coeficientes de caminho do modelo interno são apresentados na Tabela 4.

Tabela 4 Resultados de teste de significância dos coeficientes do caminho do modelo estrutural

\begin{tabular}{lccccc}
\hline & $\begin{array}{c}\text { Coeficiente } \\
\text { de caminhos }\end{array}$ & Valor de t & p-valor & Hipótese & $\begin{array}{c}\text { Conclusão sobre } \\
\text { a hipótese }\end{array}$ \\
\hline Mercado -> Rentabilidade & 0,048 & 0,643 & 0,521 & $\mathrm{H}_{1}$ & Não confirmada \\
\hline Tamanho -> Rentabilidade & 0,060 & 0,886 & 0,376 & $\mathrm{H}_{2}$ & Não confirmada \\
\hline Captação -> Rentabilidade & 0,430 & 1,665 & 0,096 & $\mathrm{H}_{3}$ & Confirmada \\
\hline Diversificação -> Rentabilidade & 0,143 & 1,725 & 0,085 & $\mathrm{H}_{4}$ & Confirmada \\
\hline Despesa Operacional -> Rentabilidade & $-0,175$ & 2,190 & 0,029 & $\mathrm{H}_{5}$ & Confirmada \\
\hline
\end{tabular}

Fonte: elaborado pelos autores

Por meio da Tabela 4, é possível observar que as hipóteses $\mathrm{H}_{1}$ e $\mathrm{H}_{2}$, referentes ao efeito das variáveis macroeconômicas do mercado e do tamanho das instituições financeiras sobre a rentabilidade, não foram confirmadas. A relação teórica (caminho) prevista entre todos os constructos não foi estatisticamente significativa, como se pode observar pelos valores da estatística t e pelo $p$-valor. Os valores são considerados estatisticamente significativos a $10 \%$ quando os $p$-valores são inferiores a 0,1. O p-valor foi de 0,521 para a $\mathrm{H}_{1}$, e de 0,376 para a $\mathrm{H}_{2}$.

Por outro lado, as hipóteses $\mathrm{H}_{3}$ e $\mathrm{H}_{4}$ (que previam um efeito positivo da captação e da diversificação, respectivamente) e a hipótese $\mathrm{H}_{5}$ (que supunha um efeito negativo das despesas operacionais) foram confirmadas. A captação apresentou um efeito interior mais forte sobre a rentabilidade $(0,430)$, enquanto que a diversificação apresentou um efeito de 0,143 . Ambos foram estatisticamente significativos a $10 \%$. Já a despesa operacional apresentou um efeito interior negativo $(-0,175)$ sobre a rentabilidade, sendo estatisticamente significativa a 5\%. Esses resultados estão em conformidade com os obtidos pelos estudos internacionais de Naceur e Omran (2011), Trujillo-Ponce (2013), Almazari (2014), Carvalho e Ribeiro (2016) e Akinkunmi (2017), bem como com os obtidos pelos estudos nacionais de Rover et al. (2011), Primo et al. (2013) e Bittencourt et al. (2017).

Além de avaliar a significância dos coeficientes dos caminhos e o nível dos valores de $\mathrm{R}^{2}$, é importante também discutir o tamanho dos efeitos $\left(\mathrm{f}^{2}\right)$. Os resultados para os valores do tamanho do efeito são apresentados na Tabela 5. 


\section{(9REUNIR}

Tabela 5 Resultados do tamanho efeito para o modelo

\begin{tabular}{ll}
\hline \multicolumn{1}{c}{ Constructos } & $\mathbf{f}^{\mathbf{2}}$ \\
\hline Captação & 0,216 \\
\hline Despesa Operacional & 0,034 \\
\hline Diversificação & 0,023 \\
\hline Mercado & 0,003 \\
\hline Tamanho & 0,004 \\
\hline \multicolumn{2}{c}{ Fonte: elaborada pelos autores }
\end{tabular}

Hair Jr. et al. (2014) explicam que ao analisar o tamanho do efeito, considera-se que os valores para $\mathrm{f}^{2}$ inferiores a 0,02 , indicam um efeito pequeno; já os valores entre 0,15 e 0,35 indicam um efeito médio, e valores superiores a 0,35 indicam um efeito grande. Diante disso, por meio da Tabela 5 é possível inferir que o constructo captação apresenta um efeito médio e os constructos despesa operacional, diversificação, mercado e tamanho apresentam um efeito pequeno sobre o constructo rentabilidade. Sendo assim, a captação das instituições financeiras bancárias (capacidade da instituição de receber depósitos à vista) apresenta um impacto maior na rentabilidade dessas instituições se for comparada com os demais constructos utilizados no modelo.

\section{CONSIDERAÇÕES FINAIS}

O presente estudo identificou alguns fatores que influenciam a rentabilidade das instituições financeiras bancárias no Brasil. A partir da aplicação da Modelagem de Equações Estruturais, as hipóteses para a captação, a diversificação e a despesa operacional foram confirmadas.

Dentre todos os constructos, a captação apresentou o efeito interior mais forte $(0,430)$ sobre a rentabilidade, sendo estatisticamente significativo a $10 \%$. Essa relação positiva indica que quanto maior for a captação (capacidade da instituição de receber depósitos à vista) maior será a rentabilidade. Já a diversificação apresentou um efeito de 0,143 e foi estatisticamente significativa a $10 \%$. A relação direta indica que um maio número de fontes alternativas de receita (tais como receitas com serviços e taxas) também está associado a uma maior rentabilidade das instituições financeiras.

Além disso, a hipótese que supunha um efeito negativo para a despesa operacional foi confirmada, identificando-se um efeito interior de -0,175 sobre a rentabilidade, estatisticamente significativo a $5 \%$. Fica evidente, assim, que as instituições financeiras devem controlar os gastos operacionais para terem uma maior rentabilidade.

Como principal contribuição gerencial deste estudo, identificou-se que a captação apresentou um $\mathrm{f}^{2}$ de 0,216 , que é considerado relativamente alto. Esse alto tamanho do efeito indica que as instituições financeiras devem focar maiores esforços na capacidade de captar depósitos à vista. Com isso, elas irão possuir um maior volume financeiro para intermediar com um menor custo, criando a possibilidade de aumentos substanciais na rentabilidade. Observou-se que os demais constructos apresentam um efeito pequeno sobre o constructo da rentabilidade. Com base nesses achados, pode-se inferir que a captação, no modelo proposto, é a variável mais importante para a rentabilidade das instituições financeiras bancárias brasileiras. 


\section{(9)REUNIR}

Por fim, observa-se que as hipóteses que supunham um efeito positivo das variáveis macroeconômicas do mercado e do tamanho das instituições financeiras sobre a rentabilidade não foram confirmadas. Assim, verificou-se que o tamanho das instituições e as variações na economia (no presente estudo, representadas pela inflação e Selic) não exerceram influência significativa para explicar a rentabilidade das instituições financeiras bancárias brasileiras no período analisado.

Vale mencionar, também, que as evidências encontradas e discutidas nesta pesquisa devem ser consideradas levando em conta os critérios de seleção da amostra (as 150 instituições financeiras no período de 2012 a 2016) e as limitações da metodologia adotada. Há a limitação das variáveis utilizadas no Modelo de Equações Estruturais, uma vez que existem outras variáveis que podem influenciar a rentabilidade e que não foram consideradas neste estudo.

Sugere-se, para pesquisas futuras, elaborar novos constructos ou complementar os constructos propostos nesse estudo com novos indicadores, visando o aumento do poder de explicação do modelo. Considerando a importância de se investigar os fatores que influenciam a rentabilidade das instituições financeiras bancárias por meio da Metodologia de Equações Estruturais, espera-se que o presente estudo fomente a discussão sobre essa temática no cenário brasileiro.

\section{REFERÊNCIAS}

AKINKUNMI, M. A. Determinants of Banks' Profitability in Nigeria: Does Relative Market Power Matter? Journal of Finance, v. 5, n. 1, p. 42-53, 2017.

ALMAZARI, A. A. Impact of internal factors on bank profitability: Comparative study between Saudi Arabia and Jordan. Journal of Applied finance and banking, v. 4, n. 1, p. 125, 2014.

ASSAF NETO, A. Mercado Financeiro. 5. ed. São Paulo: Atlas, 2003.

ASSAF NETO, A. Estrutura e análise de balanços. 10. ed. São Paulo: Atlas, 2012.

BCB - BANCO CENTRAL DO BRASIL. 50 maiores bancos e o consolidado do Sistema Financeiro Nacional. $2017 . \quad$ Disponível em: <http://www4.bcb.gov.br/top50/port/top50.asp>. Acesso em: 15 dez. 2017.

BITTENCOURT W. R.; BRESSAN, V. G. F.; GOULART, C. P.; BRESSAN, A. A.; COSTA, D. R. DE M.; LAMOUNIER, W. M. Rentabilidade em Bancos Múltiplos e Cooperativas de Crédito Brasileiros. Revista de Administração Contemporânea, v. 21, p. 22-40, 2017.

BORIO, C.; GAMBACORTA, L.; HOFMANN, B. The influence of monetary policy on bank profitability. International Finance, v. 20, n. 1, p. 48-63, 2017.

CARVALHO, J. F.; RIBEIRO, A. Fatores explicativos da rendibilidade do setor bancário: evidência empírica em Portugal. Estudos do ISCA, n. 13, 2016. 


\section{(9)REUNIR}

FIORDELISI, F. Efficiency and shareholder return in banking. International Journal of Banking, Accounting and Finance, v. 1, n. 2, p. 114-132, 2008.

FORNELL, C.; LARCKER, D. F. Evaluating structural equation models with unobservable variables and measurement error. Journal of Marketing Research, p. 39-50, 1981.

FRANCIS, M. E. Determinants of commercial bank profitability in Sub-Saharan Africa. International Journal of Economics and Finance, v. 5, n. 9, p. 134, 2013.

GIL, A. C. Como Elaborar Projetos de Pesquisa. 5. ed. São Paulo: Atlas, 2010.

HAIR JR, J. F.; HULT, G. T. M.; RINGLE, C.; SARSTEDT, M. A primer on partial least squares structural equation modeling (PLS-SEM). Sage Publications, 2014.

HAKENES, H.; SCHNABEL, I. Bank size and risk-taking under Basel II. Journal of Banking \& Finance, v. 35, n. 6, p. 1436-1449, 2011.

KIRKWOOD, J.; NAHM, D. Australian banking efficiency and its relation to stock returns. Economic Record, v. 82, n. 258, p. 253-267, 2006.

MARTINS, G. de A. Manual para elaboração de monografias e dissertações. São Paulo: Atlas, 2002.

MENDONÇA, D. J.; ALVES, J.; BENEDICTO, G. C.; CARVALHO, F. DE M.; SILVA, S. N. A. Relação entre eficiência econômico-financeira e lucratividade em instituições bancárias brasileiras. Revista de Finanças e Contabilidade da Unimep, v. 4, n. 1, p. 10-37, 2017.

NACEUR, S. B.; OMRAN, M. The effects of bank regulations, competition, and financial reforms on banks' performance.Emerging Markets Review, v. 12, n. 1, p. 1-20, 2011.

NASCIMENTO, J. C. H. B. do; MACEDO, M. A. da S. Modelagem de equações estruturais com mínimos quadrados parciais: um exemplo da aplicação do SmartPLS ${ }^{\circledR}$ em pesquisas em Contabilidade. Revista de Educação e Pesquisa em Contabilidade, v. 10, n. 3, 2016.

PRIMO, U. R., DANTAS, J. A., MEDEIROS, O. R., \& CAPELLETTO, L. R. Determinantes da rentabilidade bancária no Brasil. Base, v. 10, n. 4, 2013.

RINGLE, C. M.; SILVA, D. da.; BIDO, D. de S. Modelagem de equações estruturais com utilização do SmartPLS. REMark, v. 13, n. 2, p. 54, 2014.

ROVER, S.; TOMAZZIA, E. C.; FÁVERO, L. P. Determinantes econômico-financeiros e macroeconômicos da rentabilidade: evidências empíricas do setor bancário brasileiro.In: ENCONTRO DA ANPAD, 35, 2011, Rio de Janeiro, Anais... Rio de Janeiro: ANPAD, 2011. p. 117. 


\section{(9)REUNIR}

SUFIAN, F.; MAJID, M. Z. A. Bank efficiency and share prices in China: Empirical evidence from a three-stage banking model. International Journal of Computational Economics and Econometrics, v. 1, n. 1, p. 23-47, 2009.

TABACHNICK, B.; FIDELL L. Using multivariate statistics. New York: Harper Collins College Publishers, 1996.

TRUJILLO-PONCE, A. What determines the profitability of banks? Evidence from Spain. Accounting \& Finance, v. 53, n. 2, p. 561-586, 2013.

WHEELOCK, D. C.; WILSON, P. W. The evolution of cost-productivity and efficiency among US credit unions. Journal of Banking \& Finance, v. 37, n. 1, p. 75-88, 2013. 